\title{
Accidental severe bronchial aspiration of barium uncovers diagnosis of bronchiectasis
}

\author{
Eleanor Gerada $\cdot$ Jurgen Gerada
}

Received: 3 March 2013/ Accepted: 28 May 2013/Published online: 12 June 2013

(C) SIMI 2013

A 77-year-old man, with a history of chronic obstructive pulmonary disease (COPD) and radiotherapy-treated laryngeal malignancy diagnosed 15 years prior, presented with a 1-year history of intermittent dysphagia. As a part of his investigations, a barium swallow was obtained; but during the initial phases of the procedure, the patient aspirated the contrast agent of barium sulphate. A chest radiograph (Fig. 1a), taken few minutes after the aspiration, showed barium within the different segments and subsegments of the bronchial tree bilaterally, and uncovered features, such as irregular dilatation of the bronchial tree, non-tapering of the bronchi, luminal filling defects and lack of bronchial side branches, characteristic of bronchiectasis. This incident precipitated an exacerbation of COPD with type II respiratory failure, requiring bronchodilators, intravenous antibiotics and non-invasive ventilation using a bilevel positive airway pressure machine. On recovery, direct laryngoscopy revealed post-radiotherapy changes but no evidence of tumour recurrence. A speech and language pathology assessment using a few millilitres of water revealed weak laryngeal elevation, and immediate aspiration of water on swallowing. Given the high risk of further aspirations, advice regarding the benefits and risks of nasogastric tube insertion or percutaneous endoscopic gastrostomy tube insertion were given to the patient, but he declined both procedures. Six months following discharge, he was readmitted with an episode of food aspiration. A chest radiograph (Fig. 1b) revealed

E. Gerada $(\bowtie) \cdot$ J. Gerada

Department of Medicine, Mater Dei Hospital,

Msida MSD 2090, Malta

e-mail: egerada@gmail.com

J. Gerada

e-mail: jurgen.gerada@gmail.com residual barium in both lung bases. This event precipitated another exacerbation of the COPD and bronchiectasis, which caused the patient to succumb to the disease.

As early as the 1920s, the concept of utilizing contrast agents to aid in the diagnosis of pulmonary diseases, in particular bronchiectasis, had transpired. This involved instilling the contrast medium within the bronchial tree, by means of a catheter introduced in the trachea, and followed by the acquisition of various radiographs; a procedure known as bronchography. The initial contrast agents that were used in this regard were mainly water- and oil-based solutions. Subsequently in 1950s, barium sulphate, an inert and non-toxic agent, became a popular bronchographic medium that produced similar images as the earlier contrast agents, until the mid-1970s, when computed tomography became the gold standard imaging study for the investigation of pulmonary diseases.

Although the earlier contrast agents, as well as barium sulphate, were considered safe bronchographic media, reports of pneumonitis and even death had been described with both [1]. The instilled contrast agent was found to cause obstruction of the small bronchi, followed by atelectasis of lung tissue and pneumonitis [2]. COPD patients often developed exacerbations after such procedures, as seen in our case. To minimize these risks, after the acquisition of the radiographs, patients were asked to cough and expectorate as much contrast dye as possible in an attempt to clear the airways. However, this mechanism would have been ineffective in cases of extensive small airway filling or 'alveolarization' with the contrast medium, as the alveoli lies outside the range of the normal mechanisms (cough and ciliary action) for cleansing the bronchial tree.

The case we present demonstrates bronchographic features of bronchiectasis following accidental aspiration of 
Fig. 1 Aspiration of barium: a Chest radiograph showing barium in the segmental and sub-segmental branches of the bronchial tree of the lower lobes bilaterally, as well as in the smaller airways, producing a tree-in-bud appearance (arrow) and highlighting characteristic features of bronchiectasis. b Chest radiograph taken 6 months after the initial aspiration showing residual barium within the alveolar spaces
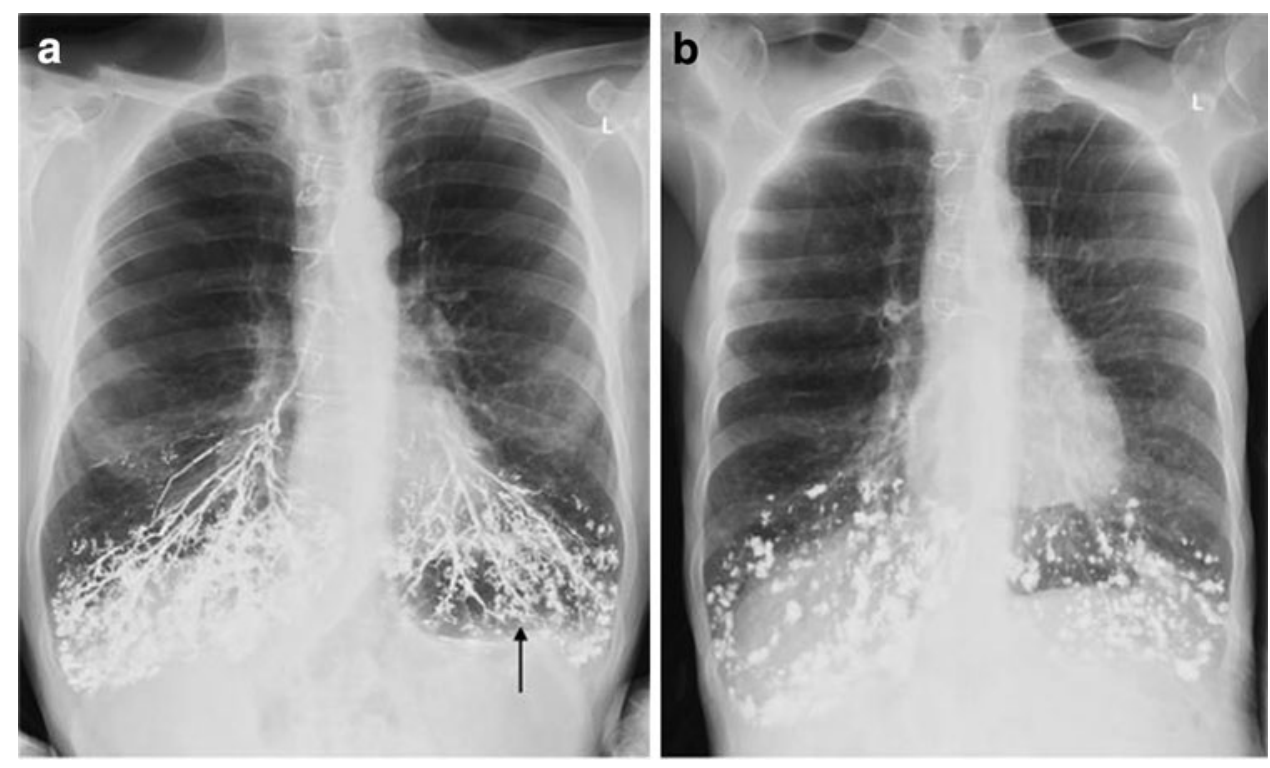

barium sulphate; features similar to the ones described by Reid et al. [3]. Apart from highlighting the patient's diagnosis, rapid distribution of barium within the bronchial tree led to alveolarization and retention of barium in the alveolar spaces, thus explaining the findings of the radiograph taken 6 months later.

When barium aspiration occurs, the volume aspirated has been reported to play an important role in the progression of aspiration [4]. When considerable amounts of barium sulphate are aspirated, leading to hypoxaemia, bronchoscopy as a therapeutic modality should be recommended to eliminate as much of the compound as possible [5]. It also facilitates collection of aspirates for microbiological analysis [5]. Our patient survived the initial insult without the use of bronchoscopy, but because of barium retention and exacerbation of COPD, he did not have enough respiratory reserve to survive the second insult (food aspiration). Thus, in massive aspiration of barium, bronchoscopy with a view to eliminate the compound should be encouraged.
Acknowledgments No funding sources were required for this manuscript.

Conflict of interest None.

\section{References}

1. Fruchter O, Dragu R (2003) A deadly examination. N Engl J Med 348:1016

2. Waldbott GL (1946) Reaction following bronchography with iodized oil. JAMA 130(9):599

3. Reid LM (1950) Reduction in bronchial subdivisions in bronchiectasis. Thorax 5:233-247

4. Katsanoulas C, Passakiotou M, Mouloudi E, Gritsi-Gerogianni N, Georgopoulou V (2007) Severe barium sulphate aspiration: a report of two cases and review of the literature. Signa Vitae 2(1):25-28

5. Tamm I, Kortsik C (1999) Severe barium sulfate aspiration into the lung: clinical presentation, prognosis and therapy. Respiration $66: 81-84$ 